\section{The self-similar rippling of leaf edges and torn}

\section{plastic sheets}

\author{
Basile Audoly, Laboratoire de modélisation en mécanique, \\ Université Pierre et Marie Curie, Paris, France
}

$P^{\text {atterns in biology are naturally appealing to physicists with a }}$ $P$ background in nonlinear sciences where structures appear spontaneously in simple, featureless systems driven out of equilibrium. How do beautiful and complex shapes like that of a flower emerge from just a simple seed? What can we learn from physics?

To some extent, the organisation of living organisms follows the same basic rules as that of complex inert systems. Some of the patterns arising in biological sciences have recently been shown to rely on the elementary principles of physics and mechanics. Steele et al., and Shipman and Newell for instance found that phyllotaxy - the spiral pattern of seeds on flowers or of leaves on a stem-could result from the buckling of a cell layer. Couder et al. showed a striking similarity between the patterns of veins on leaves, and cracks on drying gels. Goriely and Tabor, and Boudaoud investigated cell growth in analogy with inflating a balloon.

Here, we present a simple mechanical system that reproduces fascinating fractal structures observed in several biological systems: our analysis starts with a careful inspection of torn plastic packages...

Take a regular plastic sheet such as a supermarket plastic bag, make a notch at one of its edges and slowly tear the bag apart. Along the edges that are formed by tearing, a characteristic wavy pattern involving many lengthscales, shown in Fig. 1, is often observed. What is the origin of this complex pattern? Is it somehow related to the similar patterns present along the edges of some leaves and other slender biological tissues (Fig. 1)?

To address these questions, we have performed a controlled version of the above tearing experiment. Homogeneous Polyethylene sheets of thickness in the range $12-500 \mu \mathrm{m}$ were torn with a constant velocity much smaller than the typical sound speed in the material, by applying a transverse tension. A fine grid of dots printed upon the sheet surface allowed the visualization of the deformation field around the advancing tip. This visualization revealed that the sheet is irreversibly stretched within a region of the order of one centimeter around the advancing tip. Such irreversible deformations are caused by plastic flow of the material, which is triggered by the concentration of stresses near the crack tip. After a short transient following crack initiation, a steady, quasi-static regime of propagation is reached. As a result, the profile of deformation far behind the tip was found to be independent of the $x$ coordinate. This invariance makes it possible to fully characterize the irreversible deformations in the sheet after the crack has passed through by means of a single function $g(y)$. Here, $y$ denotes the coordinate perpendicular to the crack direction, that is the distance to the edge. This function $g(y)$ gives the rate of elongation of the sheet in the direction $x$ parallel to the edge, measured at a distance $y$ from this edge (see Fig. 2).
From a geometrical point of view, the tearing is a process that prepares the sheet in a new state, as the irreversible plastic flow near the crack tip redefines the "natural distances" between neighbouring points on the sheet. The origin of the rippled patterns localised near the edge can now be understood: they allow the sheet to match its edge, which has significantly been elongated by plasticity, with its undisturbed, flat profile far away from this edge. In terms of elastic energy, the flat configuration of the new elongated state is no longer a minimum of energy. To minimize its energy, the sheet has to adopt a non-planar configuration, which has fewer symmetries than a half-plane; this symmetry breaking is called buckling in the context of elasticity. While this simple argument successfully accounts for ripples, it fails to explain the complex patterns observed in the experiments: a pattern made of single wavelength, instead of many superimposed wavelengths, can as well accommodate the presence of a stretched edge. The explanation of the self-similar cascade requires more elaborate arguments, and will be elucidated below using the theory of thin elastic plates.

We now come back to the measurement of $g(y)$, which is the rate of elongation of a curve drawn parallel to the sheet edge at a distance $y$ from this edge, caused by irreversible plastic deformations. Torn sheets were placed under a profilometer to obtain the maps $z(x, y)$ of the surface. By integration of this profile, the length of the sheet in the direction $x$ parallel to the edge, measured at a fixed distance $y$ from it, could be determined; the value of this length normalized by the length of the sheet in the undisturbed flat region is $(1+g(y))$, hence the elongation profile $g(y)$, see Fig. 2 . All profiles $g(y)$ measured this way showed a monotonic increase and got steeper towards the edge; the function $g(y)$ was systematically found to be convex, $g^{\prime \prime}(y)>0$, a remark that plays an important role in the forthcoming geometric analysis. While it is possible in principle to determine this function $g(y)$ by taking

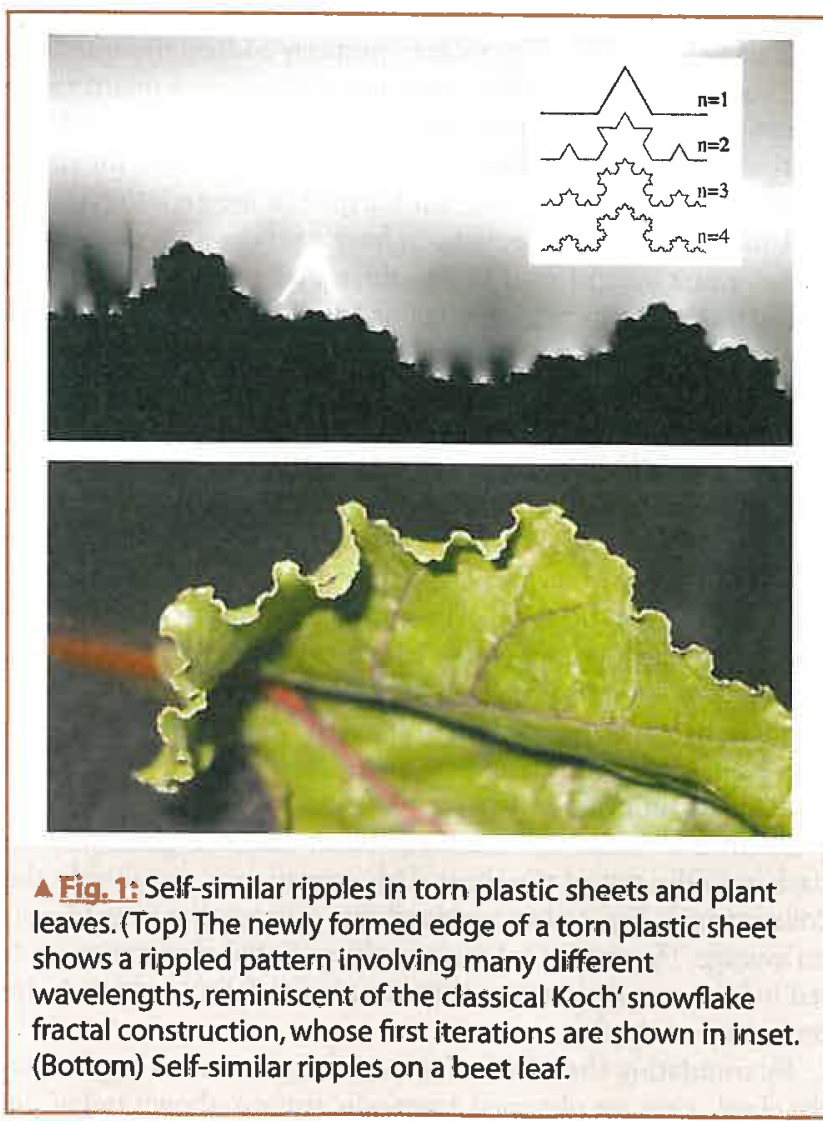


into account the coupling of plastic flow with crack propagation (theory of ductile fracture), we simply took the elongation profile $g(y)$ as a parameter of the problem, which was measured directly from the experiments.

In contrast with the 'featureless' deformation field characterized by the smooth profile $g(y)$, a typical plot of which is given in Fig. $2 \mathrm{~b}$, the relaxed sheet shapes are far from simple, see Fig. 1 . The newly formed edges show cascades of waves upon waves, where long wavelengths are visible relatively far from the edge while shorter and shorter waves are superimposed upon the formers as the edge is approached. Many of these cascades are self-similar: waves belonging to different generations have the same profile, while the dilation ratio between successive generations is a constant number in a given sheet, typically close to 3 . Up to six generation of such self-similar structures could be observed along the edge of thin $(12 \mu \mathrm{m})$ Polyethylene sheet, thus creating a fractal scaling over two and a half decades. Unlike many systems that adopt fractal geometry in a transient, unstable configuration, the fractal geometry here is the stable energy minimum of the system. It is important, however, to note that the self-similar cascades with a ratio close to 3 between successive generations are not the only one observed. By using different tearing conditions and different materials, we have obtained different cascades, some of which exhibit a ratio close to 5 between successive generations, and some which were not self-similar.

An exciting question concerns the relevance of this analysis of patterns in thin plastic sheets for the similar rippled structures that are found in naturally growing sheets such as leaves, lichens, fungi and flowers (see Fig. 1). If the growth rates are inhomogeneous on the leaf, and more active towards its edge, then an "excess of length" is generated near the edge, and similar buckling patterns can be expected. There are indeed some experimental evidences that an enhanced growth near the margins of leaves can lead to the formation of rippled leaves. However, a thorough biological work is required in order to properly address this question.

This section and the next one aim at deriving a quantitative analysis of the buckling process discussed above. The natural theoretical framework for doing so involves the equations for elastic plates, derived by Föppl and von Kàrmàn in the early $20^{\text {th }}$ century. Unfortunately, these equations have a very complex structure. To get a first insight into this problem, we present a simplified elastic model for the sheet, which is based on the theory of rods. Elastic rods are one-dimensional elastic objects, and follow much simpler equations than (two-dimensional) elastic plates. This simplified rod model has been introduced by Marder et al., and further studied by Audoly \& Boudaoud. When one cuts a thin strip of width $w$ along the edge of a sheet with a stretched edge, as shown in Fig. 3, a-c, the natural length of one side of the strip is larger than the other, as plasticity effects are more pronounced on one side than the other. Therefore, the strip cut out from the edge of the sheet spontaneously coils into a planar ring, see Fig. $3 \mathrm{c}$. Its radius of curvature is given by $R=(1+g) /\left|g^{\prime}\right|$, which is proportional to the small typical length scale $1 /\left|g^{\prime}\right|$ for the function $g(y)$. This planar ring configuration is the equilibrium shape, with minimal elastic energy, of the strip cut out from sheet. Now the problem is to cancel the cutting process, that is to glue this strip back onto the rest of the sheet. This operation is not simple: the coiled strip in Fig. $3 \mathrm{c}$ has to uncoil into a shape that is rectilinear on average. The analysis of the uncoiling of a flat elastic ring, studied below, constitutes our first simple model to approach the formation of ripples.

By simulating the effect of an unfolding moment applied on the elastic ring, we obtained a periodic pattern, shown in Fig. 3e, reminiscent of a single oscillation in the patterns shown in Fig. 1. We have reproduced this pattern experimentally using a popular toy called slinky, which has the same geometry as a planar ring in its rest configuration (Fig. 3f-g). This simple theory based on the solution of the one dimensional Kirchhoff equations for elastic rods predicts a rippling wavelength of the order of the radius of curvature of the ring, or of the small length scale $1 /\left|g^{\prime}\right|$.

Since this length scale $1 /\left|g^{\prime}(y)\right|$ decreases steeply as the edge of the torn sheet is approached, this suggests that smaller and smaller wavelength should indeed develop towards the edge. However, the self-similar character of the patterns cannot be fully understood from this rough strip model, which overlooks the two dimensional nature of in-plane stresses in the actual sheet. This calls for solving the full elastic plate problem.

Before attempting to solve the full problem, we would like to gain insight on how thin elastic sheets select their energy minimum. For isotropic elastic bodies, the material parameters are the elastic (Young's) modulus $E$ and the Poisson ratio v. The first parameter, $E$, characterizes the intrinsic stiffness of a material: a bar with cross-section area $S$ and length $L$ has a spring constant $E S / L$. The other parameter, the Poisson ratio $\mathrm{v}$, is dimensionless, and characterizes how the cross-section of the bar shrinks (or eventually expands if $\mathrm{v}<0$ ) when it undergoes simple tension. Thin sheets have two modes of deformation: bending, which only affects how the sheet is curved in the three dimensional space, and stretching, which also changes the lengths measured along the sheet. Everyday experience teaches us that it is much easier to bend a sheet, like a sheet of paper, than to stretch it. This

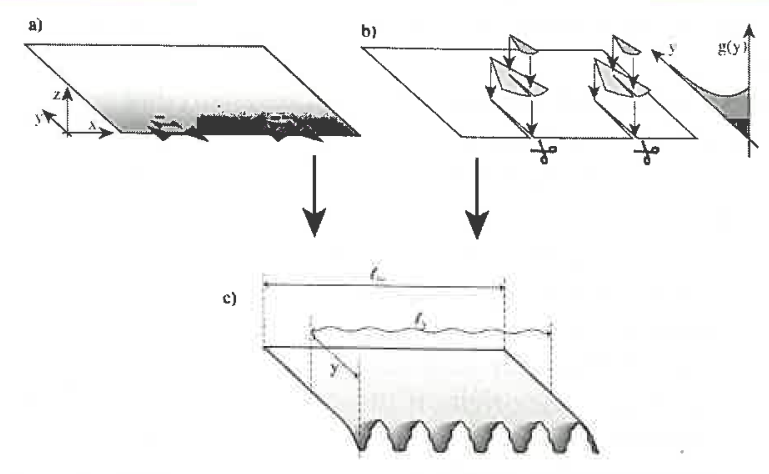

$\triangle$ Fig. 2: A torn plastic sheet is irreversibly stretched near its edge by plastic flow during crack propagation. a) As a result, residual compressive stresses are present in its planar configuration. These stresses are oriented parallel to the sheet edge and are concentrated near this edge as indicated by gray levels, b) An equivalent geometrical picture is provided by surfaces having an excess of area near an edge. Such surfaces can be constructed by inserting sequentially triangular patches into an initially flat half-plane, after Nechaev and Voiturier (incidentally, this same construction is used by fashion dressmakers to design skirts with ripples at the bottom edge, called 'godets skirts'). Distribution of the area in excess near the edge is characterized by a profile $g(y)$. c) The in-plane compressive stresses (or equivalentiy the presence of material in excess near the edge) make the sheet buckle into a complex rippled pattern. This elastic buckling allows the sheet to relax its in-plane compression. In this buckled configuration, the elongation profile $g(y)$ can be recovered by measuring the lengths $l_{y}$ of curves drawn on the surface parallel to the edge: $g(y)=\frac{l_{y}-l_{\infty}}{l_{\infty}}$. 


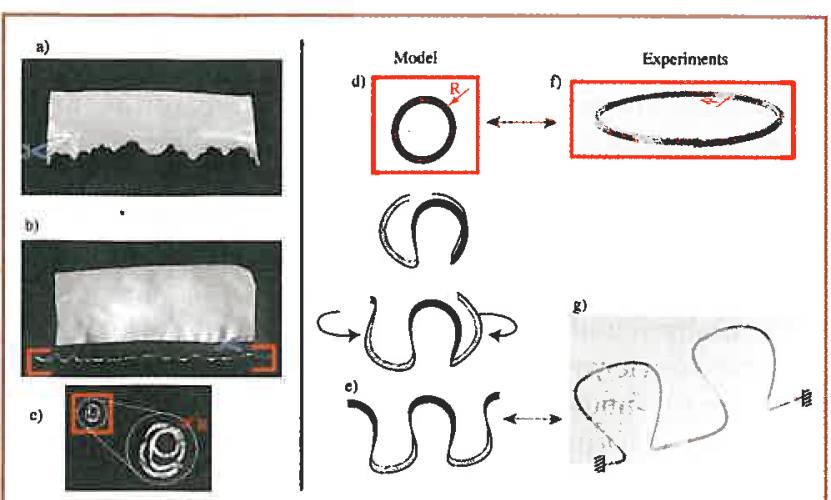

A Fig. 3: A simple elastic model for ripples based on the theory of elastic rods. (a) When one cuts a thin elastic strip along the edge of a sheet with an elongated edge, one obtains (b) a thin strip which is longer than the original sheet due to plasticity and (c) which moreover coils spontaneously into a planar ring configuration due the differential stretching of its lateral edges. By unfolding such a ring, one obtains a periodic pattern (d-e), similar to a single wavelength in the original sheet, This provides a first, simple explanation of the ripples in torn plastic sheet and plant leaves. This process is demonstrated experimentally using a well-known toy called slinky (f) unfolded using tethers ( $g$ ), not shown.

corresponds to the fact that stretching costs much more energy than bending. This key property is reflected by the typical bending energy, $E_{\mathrm{b}} \sim E h^{3}, h$ being the thickness of the plate, and the typical stretching energy $E_{\mathrm{s}} \sim E h L^{2}, L$ being the macroscopic size of the sheet; their ratio $E_{\mathrm{b}} / E_{\mathrm{s}} \sim(h / L)^{2}$ is a small number, as the aspect ratio of the thin sheet $h / L$ is typically of order $10^{-3}$. So, equilibrium configurations of a sheet favour pure bending deformations over the energetically expensive stretching ones. In other words energy minima of thin elastic sheets are very close to configurations conserving natural distances along the surface. How can we find these configurations?

It is important to notice that this last question, the existence of deformations conserving distances along a surface, is purely geometrical. To address it we must use the powerful tools of differential geometry. Within this mathematical framework, the plastic deformations, which have in some sense reset the natural distances between points on the sheet, amount to a distortion of its metric

$$
\mathrm{d} s^{2}=(1+g(y))^{2} \mathrm{~d} x^{2}+\mathrm{d} y^{2},
$$

where $x$ and $y$ are the coordinates along and perpendicular to the free edge respectively (see Fig. $2 \mathrm{a}$ ), $\mathrm{d} s$ is the infinitesimal distance between arbitrary neighbouring points separated by $\mathrm{d} x$ and $\mathrm{d} y$, and $g(y)$ is the function that characterizes how this free edge has been stretched, as defined above. When $g(y)=0$, plasticity is absent and we indeed recover the standard, flat Euclidean metric. When $g(y)>0$, equation (1) provides a rule to compute lengths of paths drawn along the surface.

There is a strong link between the metric and the curvature of a surface, which we explain now. The shape of a surface in space is described by its local curvatures. At any tangent plane to a surface, one can define two orthogonal directions, called the principal directions, along which the curvature of the surface is extremal, and has the values $c_{1}$ and $c_{2}$, called principal curvatures. The mean curvature of the surface is defined as $M=\left(c_{1}+c_{2}\right) / 2$ and its Gauss curvature as $G=c_{1} c_{2}$. For a flat plane, for instance, both curvatures vanish: $M=0$ and $G=0$; a cylinder of radius $R$ is flat in one direction and has a curvature $1 / R$ in the orthoradial direction: $M=1 /(2 \mathrm{R})$ and $G=0$; a sphere of radius $R$ (Fig. 4a) has curvature $1 / R$ in all directions: $M=1 / R$ and $G=1 / R^{2}$; also, note that for a saddle-like surface (Fig. 4b), the two principal curvatures have opposite signs: $G<0$, while $M$ can have any sign (for instance, for minimal surfaces, $M=0$ ).

There is a remarkable connection between the Gauss curvature $G$ introduced just above and the metric properties of the surface, as established by Gauss in his Theorema egregium ('remarkable theorem' in Latin). Consider a small circle of radius $r$ drawn on a sphere. This radius $r$ is measured along the surface of the sphere (and not in the three dimensional space). Using elementary geometric relations, see Fig. 5, the perimeter $p$ is easily computed as:

$$
p=2 \pi R \sin \left(\frac{r}{R}\right) \approx 2 \pi r\left(1-\frac{r^{2}}{6 R^{2}}\right)=2 \pi r\left(1-r^{2} G / 6\right),
$$

the approximation being correct for small $r / R$. The Euclidean value $2 \pi r$ of the perimeter $p$ comes in this formula with a correction that depends on the Gauss curvature G. In fact, this equation (2) is very general and gives a purely geometric interpretation of the Gauss curvature G. Knowing the metric (i.e. the distances measured along the surface between arbitrary points), one can compute the perimeters of 'circles', and hence the Gauss curvature. Depending on the sign of this Gauss curvature, the perimeters of circles are larger or smaller than in Euclidean geometry, namely smaller for so-called elliptic surfaces $(G>0)$ as a sphere, larger for hyperbolic (saddle-like) surfaces $(G<0)$, and equal to this order for parabolic surfaces $(G=0)$ like cylinders. The possibility to compute the Gauss curvature of a surface by only carrying out lengths measurements within the surface is remarkable. In fact, the 'curvature' of space-time in general relativity is defined using similar ideas.

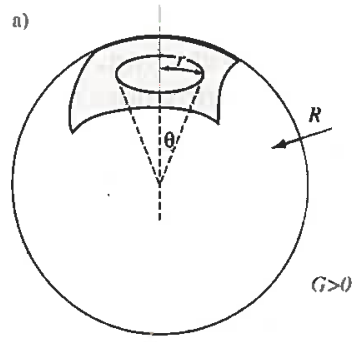

b)

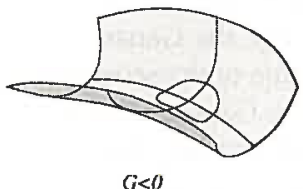

$G<0$

4 Fig. 4: The Gauss curvature $G$ is the product of the two principal curvatures of a surface and can be computed from the length of circles drawn on the surface (see Eq. 2). For elliptic surfaces such as the sphere (a), the Gauss curvature is positive as the two principal curvatures have the same sign, and small circles of a given radius have a smaller perimeter than for a flat surface - note that this radius must be measured by convention along the surface; for hyperbolic surfaces (b) with a saddle-like profile, the Gauss curvature is negative as the two principal curvatures have opposite signs, and small circles of a given radius have a larger perimeter than for a flat surface. In his Theorema egregium, Gauss showed that a length-conserving deformation of a surface also conserves the Gauss curvature. 


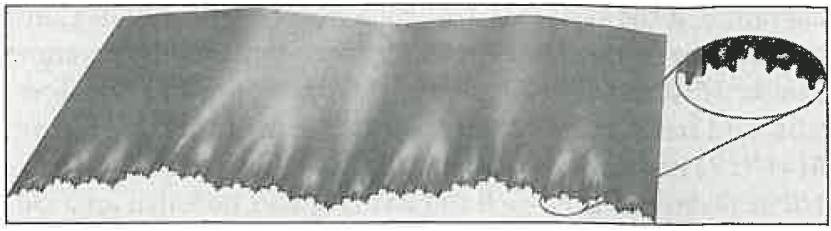

Fig.5: Numerical minimization of a plate energy, as in equation (3), yielding self-similar patterns : we have observed up to 5 generation of self-similar wrinkles in the simulations.

Plasticity, by effectively imposing a new metric on the sheet, therefore imposes the Gauss curvature of the surface in its buckled configuration. This is a strong geometrical constraint on the final state of the sheet. Calculation of the Gauss curvature of the abstract surface defined by the metric of Eq. (1) yields $G=-g$ " $(y) /(1+g(y))$. From the experimental profiles $g(y)$, we find $g^{\prime \prime}(y)>0$ and the buckled surface must therefore be hyperbolic $(G<0)$, with stronger and stronger Gauss curvature towards the edge. This predicts that all the points on our torn plastic sheet are locally saddle-like, which indeed can be easily checked on the buckling patterns.

Although it puts constraints on the buckled configuration of the sheet, geometry is not sufficient to fully predict the buckling patterns. The exact analysis of these patterns is based on the elastic energy of a sheet (Föppl-von Kàrmàn equations):

$$
\iint\left\{\frac{E h^{3}}{6\left(1-v^{2}\right)} M^{2}+\frac{E h}{8}\left[\Delta^{-1}\left(G-G_{0}\right)\right]^{2}\right\} \mathrm{d} x \mathrm{~d} y .
$$

The first term stands for the bending energy (proportional to $E h^{3}$ as stated above); its magnitude is given by the squared mean curvature $M$, which characterizes how much the surface bends. The second term (with inverse Laplacian) stands for the stretching energy (proportional to $E h$ ). The Gauss curvature $G$ gives an indirect measure of how much the surface has been stretched compared to its natural state, $G_{0}=-g$ " (for small $g$ ): as explained above, an isometric deformation of the surface, that is a deformation with no tangential stretching, does not change the Gauss curvature and consequently does not contribute to the second term in Eq. (3). Conversely, any deviation of the Gauss curvature $G$ from $G_{0}$ corresponds to a deformation of the surface that involves some stretching energy. Such deformations are penalized by the relatively large prefactor Eh in the elastic energy.

We have implemented a slightly simplified variant of these Föppl-von Kàrmàn equations, based on a justified adiabatic approximation of the elastic energy, essentially corresponding to a small slope approximation in the $y$ direction. This approximation allowed a significant gain in CPU time, something that is very valuable given the computational complexity of the minimization problem. The numerical model was further based on a Fourier decomposition of the sheet profile along the $x$ direction, that is along the torn edge, and uses 1D finite elements (i.e. polynomial interpolation) in the transverse $y$ direction. The elastic energy of the sheet was then computed symbolically as a function of the various parameters of the problem, and then minimized. This minimization led to selfsimilar buckling profiles, with cascades containing up to 5 generations of wrinkles (Fig. 5). The self-similar rippled patterns observed in torn plastic sheets and in plant leaves were therefore shown to result from the combination of two rela- tively simple ingredients: the elasticity of thin plates and a non-Euclidian metric.

As noted above, the typical lengthscale $1 / g^{\prime}$ of the metric decreases towards the edge, which qualitatively explain why superimposed modes of smaller and smaller wavelengths are generated. We now interpret the self-similarity factor $\alpha$ between two successive wavelengths in the cascade, using symmetry arguments. Consider the Fourier decomposition along $x$ of the sheet profile. As the problem under study is invariant by the up-down symmetry, only Fourier modes with the same parity are coupled. In particular, the fundamental wavelength of the cascade is only coupled to its odd harmonics. If a pattern is self-similar, the invariant dilation factor $\alpha$ must therefore map these odd harmonics onto odd harmonics. This leaves only the odd integers as possible candidates for this factor $\alpha$. The numerics shows that 3 is preferred, although 5 is sometimes only very slightly more expensive energetically. This is in good agreement with the experimentally measured factors, either $\alpha=3.2$, or $\alpha$ close to 5 , depending on the material and tearing conditions. Energetic arguments also show that the larger wavelength in the cascade is of the order of the system size (e.g. the width of the sheet), whereas the smaller wavelength, which is due to regularization by bending effects at small scales, is a combination of the thickness and the small typical length scale of the metric $g(y)$.

The simple tearing of a plastic sheet leads to complex shapes. Our theory, which addresses the buckling of a thin elastic sheet caused by an 'excess of area' near a free edge, shows that these complex shapes simply result from the minimization of the elastic energy of the sheet: by the combined effects of geometry and elasticity, simple featureless elongation profiles lead naturally to fractal-like patterns. This elastic cascade is probably at stake in the morphogenesis of ripples in leaves: it suffices that the inplane growth rate is increased near the edge of the leaf, resulting in an excess of area, for the same elastic buckling instability to induce a complex fractal pattern.

\section{References}

C. R. Steele, J. Appl. Mech. 67, 237 (2000).

P. D. Shipman and A. C. Newell, Phys. Rev. Lett. 92, 168102 (2004).

A. Goriely and M. Tabor, Phys. Rev. Lett. 90, 108101 (2003).

A. Boudaoud, Phys. Rev. Lett. 91, 018104 (2003).

S. Nechaev and R.Voiturier, J. Phys. A 34, 11069 (2001).

E. Sharon, B. Roman, M. Marder, G. S. Shin, and H. L. Swinney, Nature (London) 419, 579 (2002).

B. Audoly and A. Boudaoud, C.R. Mecanique 330, 831 (2002).

M. Marder, E. Sharon, B. Roman, and S. Smith, Europhys. Lett. 62, 498 (2003).

B. Audoly and A. Boudaoud, Phys. Rev. Lett. 91, 086105 (2003).

E. Sharon, M. Marder and H. L. Swinney, American Scientist, 92, 254-261 (2004).

U. Nath, B. C. W. Crawford, R. Carpenter, and E. Coen, Science, 299, 1404 (2003). 